\title{
NUEVOS REGISTROS DE CUCURBITACEAE PARA VARIAS REGIONES DE MEXICO
}

\author{
Rafael LiRa SAade \\ Especialidad de Postgrado de Botánica \\ Colegio de Postgraduados en Ciencias Agrícolas \\ Montecillo, Mpio. de Texcoco \\ 56230, Estado de México
}

\section{RESUMEN}

En este trabajo se registran por primera vez para varios estados de México cuatro especies de Cucurbitaceae: Cayaponia longiloba Monro (Tabasco y Veracruz), Chalema synanthera Dieterle (Sinaloa), Peponopsis adhaerens Naudin (Querétaro) y Schizocarpum palmeri Cogn. \& Rose (Veracruz). Esta última representa a su vez el primer registro del género para la porción oriental de México.

\section{ABSTRACT}

In this paper, four species of Cucurbitaceae are reported for the first time for several states of Mexico: Cayaponia longiloba Monro (Tabasco and Veracruz), Chalema synanthera Dieterle (Sinaloa), Peponopsis adhaerens Naudin (Querétaro) and Schizocarpum palmeri Cogn. \& Rose (Veracruz). The last one, also represents the first record of the genus Schizocarpum for the eastern portion of Mexico.

La revisión de materiales de herbario, en conexión con la elaboración de un inventario de la familia Cucurbitaceae de México y del estudio de la familia para la Flora del Bajío y Regiones Adyacentes (Lira, en prep.), ha permitido determinar que algunos ejemplares corresponden a nuevos registros en diferentes zonas de México, los cuales se documentan aquí. En general, se trata de especies escasamente representadas en los herbarios y cuya distribución es relativamente restringida, o bien, registros de taxones que no se incluyeron en trabajos recientes relativos a la familia o a algunos géneros.

\section{Cayaponia longiloba Monro}

Esta especie se describió recientemente con base en materiales procedentes de Oaxaca (tipo: Rovirosa 548 en NY), Chiapas y la mayor parte de Centroamérica (Monro, 1996). Al comparar ejemplares adicionales de la especie a los citados en ese trabajo con varios de los paratipos depositados en MEXU (Matuda 18710 de Chiapas y Schipp 393; 1195 de Belice), se reveló que Cayaponia longiloba también crece en los estados de Tabasco y Veracruz. El espécimen de Tabasco había sido citado por Cowan (1983) como Cayaponia alternata Cogn., nombre que aparentemente nunca se ha publicado, el cual se encuentra escrito en la etiqueta del ejemplar depositado en MEXU y que seguramente 
corresponde a una forma incorrecta de escribir C. attenuata (Hook. \& Arn.) Cogn. Los ejemplares, procedentes de Veracruz no han sido citados en el trabajo de Nee (1993).

Cayaponia longiloba se distingue de las otras dos especies del género presentes en México (C. attenuata (Hook. \& Arn.) Cogn. y C. racemosa (Mill.) Cogn.), por presentar flores y frutos de mayores dimensiones y semillas con márgenes angostos bien diferenciados. De acuerdo con Monro (1996), esta planta morfológicamente es más similar a $C$. attenuata, de la que a su vez difiere por presentar pecíolos dos veces más largos que los de esta última. De acuerdo con la información de los ejemplares revisados de Tabasco y Veracruz, Cayaponia longiloba crece en la vegetación secundaria y primaria de bosques tropicales subcaducifolios y subperennifolios, en elevaciones entre 11 y $338 \mathrm{~m}$ s.n.m. En Veracruz se le conoce con el nombre de "huevo de ratón", el cual seguramente alude a la forma y dimensiones de sus frutos.

Materiales examinados: Tabasco. Mpio. Cárdenas, carretera del Golfo km 24, 500 m al oriente del CSAT, 25.VII.1977 (hojas, flores estaminadas y flores pistiladas), J. Cantú 144 (MEXU). Veracruz. Mpio. Catemaco, Playa Azul, 31.VIII.1978 (hojas, flores pistiladas y frutos), O. H. Smith 323 (MEXU); mpio. Jamapa, región sureste de la laguna del Apompal, a $12 \mathrm{~km}$ del mpio. de Jamapa, 11.VIII.1988 (hojas y frutos), J. A. Aguilar 579 (MEXU).

\section{Chalema synanthera Dieterle}

Esta especie es endémica de la vertiente pacífica de México y es el único representante de la subfamilia Zanonioideae en esa zona del país. Fue descrita originalmente para Michoacán y Jalisco (Dieterle, 1980) y recientemente se registró por primera vez de los estados de Guerrero y Oaxaca (Lira y Torres, 1991). En esta ocasión se da a conocer su presencia en Sinaloa, lo cual sugiere que igualmente pudiera prosperar en los estados de Colima y Nayarit; de hecho, la especie ya fue incluida de manera tentativa en un estudio reciente de la familia en este último estado (Domínguez-Mariani, 1996). En Sinaloa Chalema synanthera crece sobre suelos rocosos, en bosque espinoso a 50 m s.n.m.

Materiales examinados: Sinaloa. Mpio. Culiacán, $4 \mathrm{~km}$ al $\mathrm{S}$ de Culiacán, ladera $\mathrm{S}$ del cerro del Tule, 16.X.1993 (hojas y frutos), R. Vega, A. Hernández y M. R. Vega 5012 (MEXU).

\section{Peponopsis adhaerens Naudin}

Esta especie también es endémica de México y hasta ahora sólo se conocía de unas cuantas localidades en los estados de Hidalgo, Puebla, San Luis Potosí, Veracruz y Oaxaca (Cogniaux, 1881; Lira y Torres, 1991; Nee, 1993). Ahora se documenta su presencia en el municipio de Landa de Matamoros, Querétaro, donde crece en bosques mesófilos con Quercus, Pinus, Lonchocarpus, Liquidambar, Ulmus y Dalbergia, en elevaciones que fluctúan entre 800 y 1580 m s.n.m.

Materiales examinados: Querétaro. Mpio. Landa de Matamoros, La Mora $1 \mathrm{~km}$ al NE de La Florida, 26.X.1989 (hojas, flores estaminadas y frutos), E. González 1200 (IEB, MEXU); 1.5 km SW de Río Verdito, 23.X.1989 (hojas y flores estaminadas), H. Rubio 1246 
(IEB, MEXU); $1 \mathrm{~km}$ al SE de El Pemoche, 14.VI.1989 (hojas y flores estaminadas), H. Rubio 794 (IEB, MEXU); $1.5 \mathrm{~km}$ al SE de Neblinas, 27.VI.1990 (hojas y frutos), H. Rubio 1763 (IEB, MEXU); $7 \mathrm{~km}$ al SE de Agua Zarca, sobre el camino a Pisaflores, 31.VII.1987 (hojas y flores estaminadas), J. Rzedowski 43916 (IEB, MEXU).

\section{Schizocarpum palmeri Cogn. \& Rose}

De acuerdo con la revisión más reciente de Schizocarpum (Kearns, 1992), este género incluye 11 especies distribuidas de México a Guatemala, algunas de las cuales fueron descritas como nuevas en el mencionado trabajo, sin embargo su publicación formal está aún pendiente. En dicha contribución se destaca además, la ausencia del género en la vertiente oriental de México, una aseveración que se ve apoyada por la información consignada en los pocos estudios de la familia Cucurbitaceae para esa porción del país, como el de Veracruz (Nee, 1993) y la Península de Yucatán (Lira, 1988), en donde no se registran especies de Schizocarpum.

No obstante lo anterior, en el herbario MEXU se encontraron tres ejemplares de Schizocarpum provenientes del estado de Veracruz los cuales, de acuerdo con el criterio de Kearns (1992), corresponden a S. palmeri Cogn. \& Rose. Estos especímenes proceden de dos localidades de la vertiente oriental de México ubicadas dentro del municipio de Puente Nacional, aproximadamente a 30 kilómetros de la costa. Las elevaciones en ambos sitios fluctúan entre los 100 y 150 m s.n.m. y la vegetación primaria preponderante en la zona es el bosque tropical caducifolio. En ese municipio también se han registrado varias especies cultivadas y silvestres de la familia Cucurbitaceae como Cayaponia attenuata (Hook. \& Arn.) Cogn., C. racemosa (Mill.) Cogn., Cucumis anguria L., Cucurbita argyrosperma Huber ssp. argyrosperma, C. argyrosperma ssp. sororia (L.H. Bailey) Merrick \& Bates, C. moschata (Duch. ex Lam.) Duch. ex Poir., Melothria pendula L. y Sicydium tamnifolium (H.B.K.) Cogn.

De acuerdo con Kearns (1992), la localidad más cercana a estos dos sitios para Schizocarpum palmeri es Puerto del Gato (Kearns et al. 150 en TEX), localizada 20 millas al sureste de Izúcar de Matamoros en el estado de Puebla y aproximadamente 230 a 240 kilómetros al suroeste (en línea recta) de la costa de Veracruz. Los datos proporcionados por Kearns (1992) y los antes mencionados, indican que Schizocarpum palmeri es una especie que generalmente crece en sitios con altitudes que van desde el nivel mar hasta los 800-1000 m y cuya distribución conocida comprende desde Sonora hasta Oaxaca, además de Puebla y Veracruz.

Materiales examinados: Veracruz. Mpio. Puente Nacional, Tamarindo, 4.XII.1976 (hojas, flores estaminadas y frutos), F. Ventura A. 13721; Río Escondido, 24.I.1981 (hojas y frutos), F. Ventura A. 18127; ibid., 4.XII.1981 (hojas y flores estaminadas), F. Ventura 19190 (MEXU).

\section{AGRADECIMIENTOS}

Este trabajo fue realizado con el apoyo de la Comisión Nacional para el Conocimiento y Uso de la Biodiversidad (CONABIO), mediante el proyecto "Inventario 
Florístico y Base de Datos de la Familia Cucurbitaceae de México" (CONABIO P097), conducido por el autor entre 1993 y 1995 en el Herbario Nacional (MEXU), Instituto de Biología, UNAM. Agradezco al Dr. Jerzy Rzedowski el préstamo de las colecciones de Cucurbitaceae depositadas en el herbario IEB.

\section{LITERATURA CITADA}

Cogniaux, A. 1881. Cucurbitacées. In: De Candolle A. \& C. De Candolle (eds.). Monographiae Phanerogamarum 3: 325-951.

Cowan, C. P. 1983. Listados florísticos de México I. Flora de Tabasco. Instituto de Biología, Universidad Nacional Autónoma de México, México, D.F. 123 pp.

Dieterle, J. V. A. 1980. Two new Cucurbitaceae from Mexico. Contr. Univ. Mich. Herb. 14: 69-73.

Domínguez-Mariani, A. 1996. La familia Cucurbitaceae en el Estado de Nayarit. Tesis de Licenciatura en Biología, Facultad de Ciencias, Universidad Nacional Autónoma de México. México, D.F. $124 \mathrm{pp}$.

Kearns, D. 1992. A revision of Schizocarpum (Cucurbitaceae) In: Biosystematics of Mexican Cucurbitaceae. Ph. D. Thesis, University of Texas at Austin. Austin. pp. 12-169.

Lira, R. 1988. Cucurbitaceae de la Península de Yucatán: taxonomía y etnobotánica. Tesis de Maestría en Ciencias (Ecología y Recursos Bióticos). Instituto Nacional de Investigaciones sobre Recursos Bióticos. Xalapa, Ver. 329 pp.

Lira, R. y R. Torres. 1991. Cuatro nuevos registros y una nueva especie de Cucurbitaceae para la flora de Oaxaca. Acta Bot. Mex. 16: 95-103.

Monro, A. K. 1996. A new species of Cayaponia Manso (Cucurbitaceae: Cucurbiteae) from Mesoamerica. Novon 6: 82-84.

Nee, M. 1993. Cucurbitaceae. In: Sosa, V. (ed.). Flora de Veracruz. Fascículo 74. Instituto de Ecología A.C., Xalapa, Veracruz/University of California, Riverside, CA. 133 pp. 